OPEN ACCESS

Edited by:

Olayinka Olabode Ogunleye, College of Medicine, Lagos State University, Nigeria

Reviewed by:

Ronald Kiguba

Makerere University, Uganda Andrew Lofts Gray, University of KwaZulu-Natal,

South Africa

${ }^{*}$ Correspondence:

Luciane Cruz Lopes

luciane.lopes@prof.uniso.br

Specialty section:

This article was submitted to Pharmaceutical Medicine and

Outcomes Research,

a section of the journal

Frontiers in Pharmacology

Received: 27 June 2020 Accepted: 16 December 2020 Published: 12 February 2021

Citation:

Pedroso JVM, Motter FR, Koba ST, Camargo MC, Toledo MI, Del Fiol FS, Silva MT and Lopes LC (2021)

Feasibility of De-Escalation Implementation for Positive Blood Cultures in Patients With Sepsis: A

Prospective Cohort Study.

Front. Pharmacol. 11:576849. doi: $10.3389 /$ fphar.2020.576849

\section{Feasibility of De-Escalation} Implementation for Positive Blood Cultures in Patients With Sepsis: A Prospective Cohort Study

\author{
José Victor de Miranda Pedroso ${ }^{1}$, Fabiane Raquel Motter ${ }^{1}$, Sonia Tiemi Koba ${ }^{1}$, \\ Mayara Costa Camargo ${ }^{1}$, Maria Inês de Toledo ${ }^{2}$, Fernando de Sá Del Fiol ${ }^{1}$, \\ Marcus Tolentino Silva ${ }^{1}$ and Luciane Cruz Lopes ${ }^{1 *}$ \\ ${ }^{1}$ Postgraduate Program in Pharmaceutical Sciences, University of Sorocaba, UNISO, São Paulo, Brazil, ${ }^{2}$ Posgraduate Program \\ in Tropical Medicine, University of Brasilia (UnB), Brasilia, Brazil
}

Purpose: The aim of the present study was to determine whether de-escalation guided by blood cultures for patients with a diagnosis of sepsis, severe sepsis or septic shock reduces mortality, and antimicrobial drug resistance (ADR).

Methods: A prospective, single-center, cohort study was conducted with adults admitted to the ICU with a diagnosis of sepsis, severe sepsis, or septic shock at a public hospital in Sorocaba, State of São Paulo, Brazil, from January 2013 to December 2013. We excluded patients who had negative blood cultures. Patients who had replaced the initial empirical broad-spectrum antibiotic therapy (EAT) by the antibiotic therapy guided by blood cultures were compared with those who continued receiving EAT. The outcome included mortality and antimicrobial drug resistance. We used the Cox regression (proportional hazards regression) and the Poisson regression to analyze the association between antibiotic therapy guided by blood cultures (ATGBC) and outcomes. The statistical adjustment in all models included the following variables: sex, age, APACHE II (Acute Physiology And Chronic Health Evaluation II) score and SOFA (Sequential Organ Failure Assessment) score.

Results: Among the 686 patients who were admitted to the intensive care unit, 91 were included in this study. The mean age of the patients was 52.7 years (standard deviation = 18.5 years) and $70.3 \%$ were male. EAT was replaced by ATGBC in 33 patients (36.3\%) while 58 patients $(63.7 \%)$ continued receiving EAT. Overall hospital mortality decreased from $56.9 \%$ in patients who received EAT to $48.5 \%$ in patients who received ATGBC [Hazard ratio- HR 0.44 (95\% Cl 0.24-0.82), $p=0.009$ ]. There was no association between ATGBC and ADR [HR $0.90(95 \% \mathrm{Cl} 0.78-1.03) p=0.15]$.

Conclusions: Although the early and appropriate empirical EAT is undoubtedly an important factor prognostic, ATGBC can reduce the mortality in these patients.

Keywords: sepsis, antimicrobial stewardship, antimicrobial drug resistance, intensive care units, anti-bacterial agents [MeSH] 


\section{INTRODUCTION}

Sepsis remains a serious public health problem with high morbidity and mortality. In 2017, the most recent global estimates for sepsis incidence reported 48, 9 million incident cases of sepsis and 11 million sepsis-related deaths, representing $19.7 \%$ of all global deaths (Rudd et al., 2020). Studies based on data for adults admitted to hospital show that mortality rates of sepsis range from $26.4 \%$ to $55.7 \%$ (Vincent et al., 2006; Engel et al., 2007; Machado et al., 2017; Strandberg et al., 2020; Xie et al., 2020), contributing to one-third to half of the deaths of hospitalized patients. Although therapeutic measures with considerable positive impacts have been widely emphasized, the management of sepsis in critically ill patients is challenging.

Empirical broad-spectrum antibiotic therapy (EAT) for treating sepsis, severe sepsis, and septic shock, when appropriate, reduces mortality; however, there is a risk that this treatment may expose patients to the overuse of antibiotics. It can lead to increases in antibiotic resistance, costs, and drug-associated adverse events (Martínez et al., 2020; Rhee et al., 2020). Thus, studies investigating the association between EAT and mortality among patients with sepsis have reported different findings (Paul et al., 2010). In addition, there is a relatively small number of studies that directly address the impact of the appropriate selection of antibiotic therapy in these patients (Sherwin et al., 2017).

One of the main concerns on the management of patients with sepsis is the increase in the number of infections due to multidrug-resistant pathogens which limits the treatment options. Strategies focusing on the rational use of antibiotics are essential to ensure successful outcomes and to prevent adverse antibiotic effects, and the spread of antimicrobial drug resistance (ADR) (Pradipta et al., 2013; Denny et al., 2019). In this context, antibiotic therapy guided by blood culture (ATGBC) has been proposed as a strategy to reduce unnecessary exposure to antibiotics. Even if it appears beneficial, the impact of this strategy on mortality outcomes are uncertain (Lambregts et al., 2019).

In light of these uncertainties, investigating and improving the management of antimicrobial therapy in critically ill patients with sepsis is crucial to ensure high quality and safe patient care (Mathur, 2019). Moreover, there is limited data on the impact of these strategies in patients with sepsis in low and middle-income countries such as Brazil. Therefore, this study aims to determine whether antibiotic therapy guided by blood cultures for adult patients with a diagnosis of sepsis, severe sepsis, or septic shock reduces mortality and length of hospital stay, considering relevant factors such as sex, age, and severity of sepsis.

\section{METHODS}

\section{Study Design}

A prospective, single-center, cohort study was conducted to compare mortality and length of stay in a hospital between patients who have had initial empiric therapy replaced by blood culture-guided therapy and those patients who continue receiving EAT for treatment of sepsis or sepsis severe at a public hospital in Sorocaba, State of São

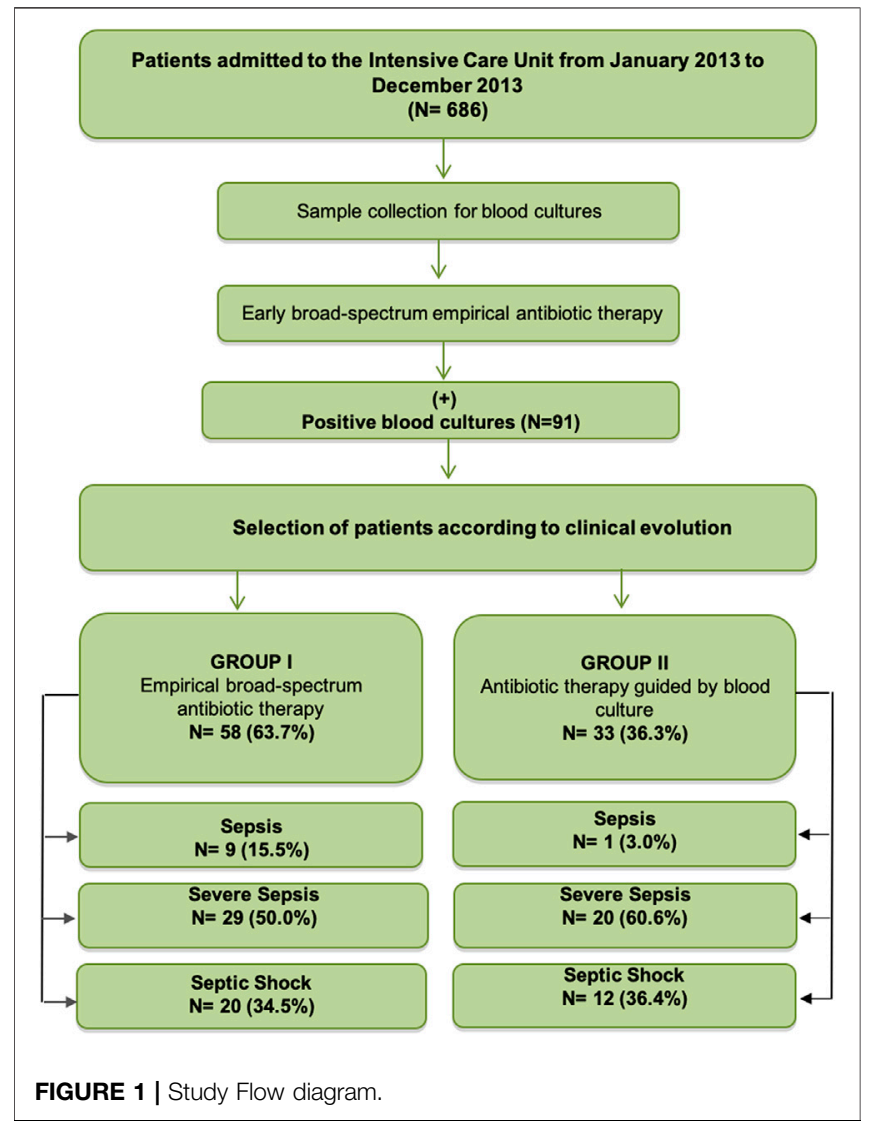

Paulo, Brazil, from January 2013 to December 2013. This study was registered at Brazilian Clinical Trials Registry (ReBEC number 98.772, UTN: U1111-1,142-1806) and it was performed in accordance with the Strengthening the Reporting of Observational Studies in Epidemiology (STROBE) guidelines (Von Elm et al., 2014).

\section{Setting}

This study was carried out in the Intensive Care Unit (ICU) of the Conjunto Hospitalar de Sorocaba (CHS), Brazil. This public hospital is part of the fourth administrative region of the State of São Paulo (Regional Health Division XVI), which serves around three million inhabitants from 48 municipalities. The CHS is a tertiary university hospital with 28 beds destined to the ICU where patients are treated by a multidisciplinary healthcare team including 30 physicians and 20 residents in clinical and surgical clinical, the technical nurse responsible and 60 nursing technicians. It is also a hospital qualified as a Teaching Institution supported by the Faculty of Medical and Health Sciences of the Pontifical Catholic University of São Paulo.

\section{Study Population}

Study subjects included hospitalized adult patients (aged $\geq 18$ years) with a diagnosis of sepsis, severe sepsis, or septic shock according to American College of Chest Physicians (ACCP) infection criteria, who were admitted to the ICU from January 
TABLE 1 | Characteristics of patients with sepsis, severe sepsis or septic shock admitted to Intensive Care Unit (ICU) in a Public Hospital according to antibiotic therapy, Sorocaba city, State of São Paulo, Brazil, 2013 ( $N=91)$.

\section{Empirical antibiotic therapy (EAT) \\ $N=58(63.7 \%)$}

\section{Antibiotic therapy guided by blood culture (ATGBC) \\ $N=33(36.3 \%)$}

\begin{tabular}{|c|c|}
\hline \multicolumn{2}{|l|}{ Age (years) } \\
\hline Mean \pm sd & $53.5 \pm 17.7$ \\
\hline \multicolumn{2}{|l|}{ Age group (years) } \\
\hline$\leq 65$ & $41(70.7)$ \\
\hline$>65$ & 17 (29.3) \\
\hline \multicolumn{2}{|l|}{ Sex } \\
\hline Men & $42(72.4)$ \\
\hline Women & $16(27.6)$ \\
\hline \multicolumn{2}{|l|}{ Ethnicity } \\
\hline Caucasian & $44(75.9)$ \\
\hline Black & $14(24.1)$ \\
\hline \multicolumn{2}{|l|}{ Severity } \\
\hline Sepsis & $9(15.5)$ \\
\hline Severe sepsis & $29(50.0)$ \\
\hline Septic shock & $20(34.5)$ \\
\hline \multicolumn{2}{|l|}{ Comorbidities } \\
\hline Presence of comorbidity & $33(56.9)$ \\
\hline Respiratory disease & $4(6.9)$ \\
\hline Cardiovascular disease & $17(29.3)$ \\
\hline Gastric disease & $7(12.0)$ \\
\hline Kidney disease & $7(12.0)$ \\
\hline Neurological disease & $2(3.5)$ \\
\hline Autoimmune disease & $2(3.5)$ \\
\hline Diabetes Mellitus & $8(13.8)$ \\
\hline Malignancy & $5(8.6)$ \\
\hline Coma on admission to the ICU & $40(68.9)$ \\
\hline Ventilator-associated pneumonia & $13(22.4)$ \\
\hline \multicolumn{2}{|l|}{ Apache ॥ } \\
\hline First day of ICU admission Mean \pm sd & $24.8 \pm 7.4$ \\
\hline Last observation ${ }^{\mathrm{C}}$ Mean \pm sd & $20.6 \pm 9.9$ \\
\hline \multicolumn{2}{|l|}{ SOFA } \\
\hline First day of ICU admission Mean \pm sd & $8.4 \pm 3.7$ \\
\hline Last observation ${ }^{\mathrm{C}}$ Mean \pm sd & $5.6 \pm 4.1$ \\
\hline \multicolumn{2}{|c|}{ Length of hospitalization before the admission to ICU } \\
\hline Mean \pm sd & $7.3 \pm 10.9$ \\
\hline Median (range) & $2.5(0-58)$ \\
\hline
\end{tabular}

$51.9 \pm 18.3$

$23(69.7)$

$10(30.3)$

$25(75.7)$

8 (24.2)

28 (84.8)

5 (15.1)

1 (3.0)

$20(60.6)$

12 (36.4)

20 (60.6)

1 (3.0)

8 (13.8)

2 (6.0)

6 (18.2)

2 (6.0)

2 (6.0)

5 (15.2)

2 (6.0)

22 (66.6)

11 (33.3)

$24.1 \pm 5.9$

$23.5 \pm 6.8$

$7.5 \pm 2.9$

$6.0 \pm 3.2$

$5.6 \pm 8.8$

$2.0(0-35)$ $0.68^{\mathrm{a}}$

p > Value

$1.00^{\mathrm{b}}$

$0.42^{b}$

$0.80^{b}$

$0.29^{b}$

$0.82^{b}$

$1.00^{\mathrm{b}}$

$0.63^{\mathrm{b}}$

$0.47^{b}$

$0.53^{b}$

$0.61^{b}$

$0.61^{b}$

$0.99^{b}$

$1.00^{\mathrm{b}}$

$0.81^{b}$

$0.54^{b}$

$0.83^{\mathrm{a}}$

$0.71^{a}$

$0.44^{a}$

at test.

${ }^{b}$ Fisher's exact test.

${ }^{c}$ Last observation carried on before death or before ICU discharged.

2013 to December 2013. All patients who had positive results in at least two blood culture were included in order to avoid errors related to colonization/contamination of surgical sites.

\section{Definitions}

Blood samples, secretion cultures (according to their origin), and blood cultures (at least three different sites) were collected for routine admission exams according to the protocols adopted by the CHS.

Blood cultures were performed by an automated process using the Bact-Alert system manufactured by bioMérieux, Inc (100 Rudolph Wtreet, Durham, NC 27712).

EAT was defined as an initial antibiotic treatment before the microorganism was identified and antimicrobial susceptibility test results were obtained. EAT was initiated for all patients included. After the result of blood cultures, the subjects were classified into two groups, according to clinical evolutionary criteria (Bone et al., 1992). Patients who were progressing satisfactorily remained on broad-spectrum EAT and those who did not show clinical improvement had their therapy replaced by an antibiotic therapy guided by blood culture which was based on blood culture and antibiotic susceptibility results.

Clinical and laboratory follow-up was carried out by attending physicians and infectious disease specialists, with an adaptation to the most specific antimicrobial therapy (smaller or larger generation), if possible for monotherapy and a shorter period of therapy.

\section{Data Collection}

We developed a software in the Access platform in order to collect data from patients included in this study. This software was divided into six stages of completion: patient's registration, symptoms, clinical and laboratory results, antibiogram, 
TABLE 2 | Microorganisms isolated in patients with sepsis. severe sepsis or septic shock obtained on admission to Intensive Care Unit (ICU) in a Public Hospital according to antibiotic therapy, Sorocaba city, State of São Paulo, Brazil, 2013 ( $N=91)$.

\begin{tabular}{|c|c|c|c|c|c|}
\hline \multirow[t]{2}{*}{ Microorganisms } & \multicolumn{2}{|c|}{$\begin{array}{l}\text { Empirical antibiotic therapy (EAT) } \\
\qquad N=58\end{array}$} & \multicolumn{2}{|c|}{$\begin{array}{l}\text { Antibiotic therapy guided by blood } \\
\text { cultures (ATGBC) } N=33\end{array}$} & \multirow[t]{2}{*}{$p$ value } \\
\hline & Total & $\% \mathbf{M R}^{\mathbf{a}}$ & Total & $\% \mathbf{M R}^{\mathbf{a}}$ & \\
\hline Gram-positive & $44(75.8)$ & & $29(87.9)$ & & $0.18^{\mathrm{b}}$ \\
\hline Enterococcus spp & $9(20.4)$ & $1(2.2)$ & $7(24.1)$ & $1(3.4)$ & \\
\hline Staphylococcus sp coag neg & $28(63.6)$ & $26(59.0)$ & $21(72.4)$ & 17 (58.6) & \\
\hline Staphylococcus aureus & 5 (11.3) & $1(2.2)$ & $6(20.6)$ & 3 (10.3) & \\
\hline Streptococcus pneumoniae & $0(0.0)$ & $0(0.0)$ & $1(3.4)$ & $0(0.0)$ & \\
\hline Gram-negative & $26(44.8)$ & & $13(39.4)$ & & $0.83^{\mathrm{b}}$ \\
\hline Enterobacter aerogenes & $9(34.6)$ & $4(15.4)$ & $3(23.1)$ & $2(15.4)$ & \\
\hline Escherichia coli & $2(7.7)$ & $1(3.8)$ & $1(7.7)$ & $0(0.0)$ & \\
\hline Klebsiella pneumoniae & $9(34.6)$ & $4(15.3)$ & $5(38.4)$ & $3(23.0)$ & \\
\hline Morganella morganii & $1(3.8)$ & $0(0.0)$ & $1(7.7)$ & $0(0.0)$ & \\
\hline Pseudomonas aeruginosa & $6(23.0)$ & $3(11.5)$ & $3(23.0)$ & $2(15.3)$ & \\
\hline Serratia marcescens & $0(0.0)$ & $0(0.0)$ & $1(7.7)$ & $1(7.7)$ & \\
\hline Acinetobacter baumannii & $12(46.1)$ & $11(42.3)$ & $6(46.1)$ & $6(46.1)$ & \\
\hline Citrobacter freundii & $0(0.0)$ & $0(0.0)$ & 2 (15.3) & $1(7.7)$ & \\
\hline Citrobacter koseri & $1(3.8)$ & $0(0.0)$ & $0(0.0)$ & $0(0.0)$ & \\
\hline Burkholderia cepacia & $0(0.0)$ & $0(0.0)$ & $1(7.7)$ & $0(0.0)$ & \\
\hline
\end{tabular}

${ }^{a} M R=$ Multi-resistance

${ }^{b}$ Fisher's exact test.

comorbidities, list of antibiotics. Data were collected prospectively, and patients were followed up until death or until hospital discharge. All data collected were confirmed by two specialists (José Victor Miranda Pedroso and Eduardo Leite Croco) and were cross-checked with laboratory information which was obtained directly from the laboratory's database.

\section{Outcomes}

The primary outcome was hospital mortality. The secondary outcome was an antimicrobial drug resistance. Antimicrobial drug resistance was defined as the ability of microorganisms, especially bacteria, to resist or to become tolerant to chemotherapeutic agents, antimicrobial agents, or antibiotics. This resistance may be acquired through gene mutation or foreign DNA in transmissible plasmids.

\section{Variables}

The following data were collected prospectively for all included patients from the time of admission into the ICU: age, sex, length of hospital stay, the origin of infection, chronic diseases, and comorbidities, type of sepsis (sepsis, severe sepsis, and septic shock), need for mechanical ventilation, hemodialysis, surgical interventions, drainage, blood cultures, and antibiotic therapy. The severity of the disease was assessed using the APACHE II index (Acute Physiology And Chronic Health Evaluation II). The development of organ failures was accompanied by the SOFA index (Sequential Organ Failure Assessment). Both APACHE II index and SOFA were estimated on the first day of ICU admission and the last observation carried on before death or before ICU discharged.

\section{Statistical Analysis}

Analyses were performed using Stata v.12 (Stata Corp., College Station, TX, United States). For descriptive statistics, we examined characteristics among patients who received EAT vs. patients who received therapy guided by blood culture. Based on distribution data we used unpaired $t$-test, chi-squared test, or Fisher's exact test.

The data were described using proportions and contingency tables for categorical variables and measures of central tendency and dispersion for continuous variables (age, Apache II, SOFA).

Hazard ratios (HRs) and 95\% confidence intervals (CIs 95\%) were obtained using Cox regression (proportional hazards regression) in order to estimate the effect of therapy directed by blood culture on mortality and hospital mortality. With regard to acquired antimicrobial drug multiresistance, the relative risk and CIs 95\% were calculated using Poisson regression. The statistical adjustment in all models included the following variables: sex, age, APACHE score, and SOFA score. All tests with $p$-values $<0.05$ were considered statistically significant.

\section{RESULTS}

During the study period, 686 adults were admitted to the ICU, 91 eligible patients with sepsis, severe sepsis, or septic shock were included, according to the inclusion and exclusion criteria (Figure 1). The mean age of the patients was 52.7 years (standard deviation $=18.5$ years) and $70.3 \%$ were male. They were classified into two groups: the broad-spectrum EAT group (58 patients) and the ATGBC group (33 patients).

Characteristics of the patients included in this study according to antibiotic therapy received are detailed in Table 1. There was no significant difference between patients who received EAT and the ATGBC with respect to sociodemographic characteristics such as age, sex, and ethnicity. 
TABLE 3 | The most common Multidrug Resistant Bacteria isolated in the blood cultures of patients with sepsis, severe sepsis or septic shock admitted to Intensive Care Unit (ICU) in a Public Hospital, Sorocaba city, State of São Paulo, Brazil, $2013(N=91)$

\begin{tabular}{|c|c|c|c|c|c|c|c|c|c|c|}
\hline \multirow[b]{2}{*}{ Antibiotic } & \multicolumn{2}{|c|}{ Enterobacter aerogenes } & \multicolumn{2}{|c|}{ Staphylococcus sp coag neg } & \multicolumn{2}{|c|}{ Klebsiella pneumoniae } & \multicolumn{2}{|c|}{ Pseudomonas aeruginosa } & \multicolumn{2}{|c|}{ Acinetobacter baumanii } \\
\hline & $\mathrm{Na}$ & $\mathrm{Rb}(\%)$ & $\mathrm{Na}$ & $\mathrm{Rb}(\%)$ & $\mathrm{Na}$ & $\mathrm{Rb}(\%)$ & $\mathrm{Na}$ & $\mathrm{Rb}(\%)$ & $\mathrm{Na}$ & $\mathrm{Rb}(\%)$ \\
\hline \multicolumn{11}{|l|}{ Penicillins } \\
\hline Penicillin G & - & - & 54 & $51(94.4)$ & - & - & - & - & - & - \\
\hline Oxacillin & - & - & 56 & 54 (96.4) & - & - & - & - & - & - \\
\hline Ampicillin & 12 & $12(100.0)$ & - & - & 16 & $16(100.0)$ & - & - & 4 & $4(100.0)$ \\
\hline Amoxicillin & 10 & $10(100.0)$ & - & - & 14 & $9(64.3)$ & - & - & - & - \\
\hline Piperacillin & 13 & 11 (84.6) & - & - & 16 & $11(68.7)$ & 11 & $7(70.7)$ & 18 & $17(94.4)$ \\
\hline \multicolumn{11}{|l|}{$\begin{array}{l}\text { Cephalosporins } \\
\text { 1st Generation }\end{array}$} \\
\hline Cephalothin & 12 & $12(100.0)$ & - & - & 15 & 11 (73.3) & - & - & - & - \\
\hline \multicolumn{11}{|l|}{ 2nd Generation } \\
\hline cefoxitin & 12 & $12(100.0)$ & - & - & 17 & $12(70.6)$ & - & - & - & - \\
\hline \multicolumn{11}{|l|}{ 3rd Generation } \\
\hline ceftazidime & 12 & $10(83.3)$ & - & - & 15 & $10(66.6)$ & - & - & - & - \\
\hline ceftriaxone & 12 & 10 (83.3) & & & 12 & $11(91.6)$ & 11 & $8(72.7)$ & 18 & $18(100.0)$ \\
\hline \multicolumn{10}{|l|}{ 4th Generation } & $16(94.1)$ \\
\hline \multicolumn{11}{|l|}{ Carbapenems } \\
\hline Meropenem & 11 & $0(0.0)$ & - & - & 15 & $1(11.2)$ & 11 & 7 (63.6) & 18 & $15(83.3)$ \\
\hline Imipenem & 12 & $0(0.0)$ & - & - & 15 & $0(0.0)$ & 11 & 7 (63.6) & 18 & $14(77.7)$ \\
\hline Ertapenem & 12 & $1(8.3)$ & - & - & 15 & $0(0.0)$ & - & - & - & - \\
\hline \multicolumn{11}{|l|}{ Quinolones } \\
\hline Ciprofloxacin & 12 & $6(50.0)$ & 51 & 45 (88.2) & 16 & $10(62.5)$ & 11 & $8(72.7)$ & 18 & 16 (88.8) \\
\hline \multicolumn{11}{|l|}{ Aminoglycosides } \\
\hline Amikacin & 12 & $3(25.0)$ & - & - & 16 & $2(12.5)$ & 10 & $6(60.0)$ & 18 & $14(77.7)$ \\
\hline Gentamycin & 12 & $9(75.0)$ & 56 & $49(87.5)$ & 16 & $7(43.8)$ & 11 & $8(72.7)$ & 18 & 16 (88.8) \\
\hline Tobramycin & - & - & - & - & - & - & 11 & 7 (63.6) & 18 & $16(88.8)$ \\
\hline \multicolumn{11}{|l|}{ Sulfonamides } \\
\hline Sulfamethoxazole & 11 & $7(63.6)$ & 54 & $44(81.5)$ & 16 & $10(62.5)$ & - & - & 18 & $16(88.8)$ \\
\hline \multicolumn{11}{|l|}{ Macrolides } \\
\hline Erythromycin & - & - & 54 & 51 (94.4) & - & - & - & - & - & - \\
\hline \multicolumn{11}{|l|}{ Chloramphenicol } \\
\hline Chloramphenicol & 12 & $7(58.3)$ & 55 & $32(58.2)$ & 16 & $8(50.0)$ & - & - & - & - \\
\hline \multicolumn{11}{|l|}{ Glycopeptides } \\
\hline Vancomycin & - & - & 54 & $1(1.8)$ & - & - & - & - & - & - \\
\hline \multicolumn{11}{|l|}{ Lincosamides } \\
\hline Clindamycin & - & - & 52 & 48 (92.3) & - & - & - & - & - & - \\
\hline \multicolumn{11}{|l|}{ Monobactams } \\
\hline Aztreonam & 12 & 10 (83.3) & - & - & 14 & 11 (78.6) & 11 & $8(72.7)$ & 18 & $17(94.4)$ \\
\hline \multicolumn{11}{|l|}{ Polymyxins } \\
\hline Polymyxin B & - & - & - & - & - & - & 10 & $1(10.0)$ & 18 & $1(5.5)$ \\
\hline \multicolumn{11}{|l|}{ Oxazolidinones } \\
\hline Linezolid & - & - & 56 & $0(0.0)$ & - & - & - & - & - & - \\
\hline
\end{tabular}

${ }^{a} N=$ number of positive blood cultures where specific antibiotic was tested.

${ }^{\circ} R=$ number of positive blood cultures where the causative agent was resistant to the antibiotic. 
TABLE 4 | Number of days of antibiotic use in patients with sepsis, severe sepsis or septic shock admitted to Intensive Care Unit (ICU) in a Public Hospital according to antibiotic therapy, Sorocaba city, State of São Paulo, Brazil, 2013 ( $N=91)$.

\begin{tabular}{|c|c|c|c|c|}
\hline \multirow[t]{2}{*}{ Types of antibiotic } & \multicolumn{2}{|c|}{ Empirical antibiotic therapy (EAT) } & \multicolumn{2}{|c|}{ Antibiotic therapy guided by blood culture (ATGBC) } \\
\hline & $\mathbf{N}=58(\%)$ & Number of days Mean \pm sd & $\mathbf{N}=33(\%)$ & Number of days Mean \pm sd \\
\hline Penicillins & $22(37.9)$ & & $22(66.6)$ & \\
\hline Penicillin G & $0(0.0)$ & $0.0 \pm 0.0$ & $1(3.0)$ & $8.0 \pm 0.0$ \\
\hline Oxacilin & $1(1.7)$ & $12.0 \pm 3.4$ & $3(9.1)$ & $3.0 \pm 1.5$ \\
\hline Amoxicilin & $0(0.0)$ & $0.0 \pm 0.0$ & 1 (3.3) & $2.0 \pm 0.0$ \\
\hline Piperacilin + Tazobactam & $21(36.2)$ & $10.4 \pm 5.3$ & $17(51.5)$ & $10.5 \pm 3.5$ \\
\hline Cephalosporins & $39(67,1)$ & & $24(75.7)$ & \\
\hline \multicolumn{5}{|l|}{ 1st Generation } \\
\hline Cefazolin & $11(18.9)$ & $7.6 \pm 4.2$ & $3(9.1)$ & $1.6 \pm 0.8$ \\
\hline \multicolumn{5}{|l|}{ 2nd Generation } \\
\hline Cefuroxime & $1(1.7)$ & $1.0 \pm 0.0$ & $0(0.0)$ & $0.0 \pm 0.0$ \\
\hline \multicolumn{5}{|l|}{ 3rd Generation } \\
\hline Ceftazidime & $0(0.0)$ & $0.0 \pm 0.0$ & $1(3.0)$ & $4.9 \pm 0.0$ \\
\hline Ceftriaxone & $20(34.5)$ & $8.15 \pm 2.1$ & $10(30.3)$ & $5.6 \pm 2.5$ \\
\hline \multicolumn{5}{|l|}{ 4th Generation } \\
\hline cefepime & 7 (12.0) & $7.1 \pm 3.0$ & $11(33.3)$ & $5.4 \pm 3.5$ \\
\hline Carbapenems & $18(31.0)$ & & $19(57.6)$ & \\
\hline Meropenem & $9(15.5)$ & $15.2 \pm 1.3$ & $9(27.3)$ & $10.4 \pm 4.1$ \\
\hline Imipenem & $5(8.6)$ & $10.6 \pm 3.5$ & $6(18.2)$ & $24.6 \pm 2.0$ \\
\hline Ertapenem & $4(6.8)$ & $11.25 \pm 2.8$ & $4(12.1)$ & $8.5 \pm 3.5$ \\
\hline Quinolones & $6(10.3)$ & & $1(3.0)$ & \\
\hline Ciprofloxacin & $5(8.6)$ & $7.0 \pm 2.6$ & $0(0.0)$ & $0.0 \pm 0.0$ \\
\hline Levofloxacin & $0(0.0)$ & $0.0 \pm 0.0$ & $1(3.0)$ & $5.0 \pm 0.0$ \\
\hline Moxiflocin & $1(1.7)$ & $3.0 \pm 0.0$ & $0(0.0)$ & $0.0 \pm 0.0$ \\
\hline Aminoglycosides & $5(8.6)$ & & $5(15.2)$ & \\
\hline Amikacin & $4(6.8)$ & $7.5 \pm 2.2$ & $4(12.1)$ & $9.7 \pm 1.5$ \\
\hline Gentamycin & $1(1.7)$ & $11.0 \pm 0.0$ & $1(3.0)$ & $21.0 \pm 0.0$ \\
\hline Sulfonamides & $3(5.2)$ & & $3(9,1)$ & \\
\hline Sulfamethoxazole & $2(3.4)$ & $14.0 \pm 0.0$ & $2(6.1)$ & $3.0 \pm 0.0$ \\
\hline Sulfadiazine & $1(1.7)$ & $7.0 \pm 0.0$ & $1(3.0)$ & $36.0 \pm 0.0$ \\
\hline Macrolides & $2(3.4)$ & & $2(6.1)$ & \\
\hline Rifampicin & $2(3.4)$ & $17.5 \pm 1.2$ & $2(6.1)$ & $23.0 \pm 0.0$ \\
\hline Amphenicols & $1(1.7)$ & & $2(6.1)$ & \\
\hline Chloramphenicol & $1(1.7)$ & $1.0 \pm 0.0$ & $2(6.1)$ & $0.0 \pm 0.0$ \\
\hline Glycylcyclines & $1(1.7)$ & & $1(3.0)$ & \\
\hline Tigecycline & $1(1.7)$ & $19.0 \pm 0.0$ & $1(3.0)$ & $21.0 \pm 0.0$ \\
\hline Glycopeptides & 19 (32.8) & & 30 (90.9) & \\
\hline Vancomycin & $18(31.0)$ & $13.6 \pm 3.1$ & 30 (90.9) & $14.0 \pm 5.2$ \\
\hline Teicoplanin & $1(1.7)$ & $1.0 \pm 0.0$ & $0(0.0)$ & $0.0 \pm 0.0$ \\
\hline Lincosamides & $10(17.2)$ & & $5(15.2)$ & \\
\hline Clindamycin & $10(17.2)$ & $8.3 \pm 2.4$ & $5(15.2)$ & $11.0 \pm 3.5$ \\
\hline Polymyxins & $3(5.1)$ & & 7 (21.2) & \\
\hline Polymyxin B & $3(5.1)$ & $8.6 \pm 1.2$ & $7(21.2)$ & $9.8 \pm 2.5$ \\
\hline Oxazolidinones & $1(1.7)$ & & $3(9.1)$ & \\
\hline Linezolid & $1(1.7)$ & $18.0 \pm 0.0$ & $3(9.1)$ & $12.0 \pm 2.0$ \\
\hline
\end{tabular}

Furthermore, statistically significant differences were not detected in the proportion of comorbidities, coma $(p=0.81)$, pneumonia associated with mechanical ventilation $(p=0.54)$ and in the median values of Apache II $(p=0.83)$ and SOFA $(p=0.71)$ and length of hospital stay before the ICU $(p=0.44)$ between the two groups.

The frequency of identified pathogens in the blood cultures according to antibiotic therapy received are presented in Table 2. Gram-positive was the most common in both types of antibiotic therapy $(75.8 \%$ of patients with EAT vs. $87.9 \%$ of patients with ATGBC therapy, $p=0.18)$. Staphylococcus sp coag neg $(63.6 \%)$ was the most frequently Gram-positive microorganism isolated, followed by Enterococcus spp. (34.6\%). Among Gram-negative microorganisms, Acinetobacter baumannii (46.1\%) was the most commonly isolated, followed by Klebsiella pneumonia (34.6\%) and Enterobacter erogenes (34.6\%).

The most frequent multidrug-resistant bacteria isolated in critically ill patients were Staphylococcus sp, followed by Acinetobacter baumanii and Klebsiella pneumoniae (Table 3). Oxacillin-resistant Staphylococcus sp and Penicillin G-resistant Staphylococcus sp are the most commonly identified.

Table 4 shows the antibiotics and number of days used according to intervention adopted. Piperacillin (36.2\%), ceftriaxone (34.5\%) and vancomycin $(31.0 \%)$ were the antibiotics most used in EAT. 


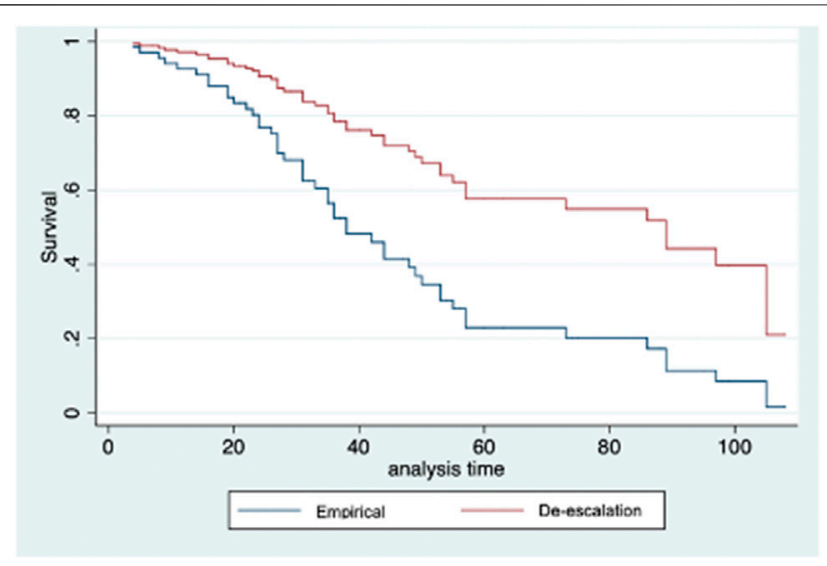

HR - Hazard Ratio; ‘Adjusted for sex, age, Apache and Sofa.

FIGURE 2 | Survival in individuals with sepsis, severe sepsis or septic shock according to the length of hospital stay. Sorocaba city, State of São Paulo, Brazil 2013.

About $90 \%$ of ATGBC patients used vancomycin, followed by piperacillin (51.5\%) and cefepime (33.3\%).

\section{Mortality}

In the study sample of 91 individuals, 49 deaths were reported, resulting in a mortality rate of $53.8 \%$. Mortality for patients who continued receiving EAT was $56.9 \%$ vs. $48.5 \%$ for patients receiving ATGBC. Figure 2 presents a survival analysis for patients during hospitalization. The hospital mortality rate of patients receiving ATGBC was statistically lower than the mortality rate of patients who continued receiving empirical treatment after adjustment for sex, age, APACHE II, and SOFA [HR 0.44 (IC 95\% 0.24 0.82), $p=0.009$ ] (Table 5).

\section{Antimicrobial Drug Resistance}

The prevalence of ADR was high in both types of Antimicrobials therapy $(93.5 \%$ ATGBC vs. $84.5 \%$ EAT). There was no association between ATGBC and ADR [HR 0.90 (95\% CI $0.78-1.03) p=0.15]$ (Table 5).

\section{DISCUSSION}

Although the safety and effectiveness of the strategy of deescalation in the use of antibiotics in severe sepsis and septic shock were questioned in a previous systematic review (Silva et al., 2013), this prospective cohort study verified that the substitution of the EAT by an ATGBC increased survival of patients with sepsis when compared to the continuation of EAT, after adjusting for potentially confounding factors such as age, sex, APACHE. Currently, The Surviving Sepsis Campaign recommends that the antibiotic spectrum should be narrowed as soon as causative pathogens and their antibiotic susceptibility profile is available. In addition, some observational studies (Garnacho-Montero et al., 2014; Tabah et al., 2016) and a meta-analysis (Gutiérrez-Pizarraya et al., 2017) have also found that de-escalation therapy was associated with lower mortality rates in patients with severe sepsis and septic shock. It is important to highlight that although there is no consensus regarding the definition of de-escalation, switching from a broad to narrow therapy based on culture data constitutes one important strategy of this process (Tabah et al., 2016).

In this study, the alteration of initial empirical therapy was applied in one-third of patients. Although the severity of the disease did not influence the decision to use the ATGBC, some physicians may be reluctant to switch from a broad-spectrum antibiotic to narrow therapy when caring for patients with sepsis, especially when the infections are caused by multidrug-resistant bacteria. Furthermore, the applicability of antimicrobial therapy to infections caused by multidrug-resistant pathogens where the use of combined broad-spectrum therapy is recommended by most specialists, makes this choice very difficult to be adopted (Kollef et al., 2011).

Gram-positive bacteria were the most frequently involved microorganisms. These findings are consistent with the literature (Martin et al., 2003). Staphylococcus sp and Acinetobacter baumannii were the most prevalent multidrugresistant bacteria. This result is similar to other studies about infections in Brazil (De Oliveira et al., 2012) and in other countries (Garnacho-Montero and Amaya-Villar, 2010; Teerawattanapong et al., 2018).

Despite the presence of ESKAPE organisms, the alteration of broad-spectrum empirical therapy by the narrow therapy did not influence the emergence of multidrug resistance pathogens. Although de-escalation is often presented as an effective strategy to reduce multidrug resistance, previous observational study (De Bus et al., 2016) and randomized clinical trial (Leone et al., 2014) found that the de-escalation did not affect the emergence of multidrug-resistant (MDR) pathogens. Only one study that evaluated the carbepen de-escalation has found significant differences in resistant Acinetobacter spp. colonization (Lew et al., 2015). According to De Waele et al. (2020) other studies with large numbers of patients are probably required to evaluate the impact of de-escalation on ADR in ICU patients.

In 2016, the new definitions of sepsis and septic shock have changed. Sepsis is now defined as life-threatening organ dysfunction caused by a dysregulated host response to infection. This definition uses the Sequential Organ Failure Assessment (SOFA $\geq 2$ ) score to discriminate sepsis from uncomplicated infection, replacing SIRS criteria that were criticized for being inaccurate. SIRS criteria are present in many hospitalized patients, including those who never develop infection (Singer et al., 2016). Although our study followed the criteria established in 2001, all patients included in this study had SOFA $\geq 2$. In addition, we only included patients with at least two positive blood cultures in order to ensure the diagnosis of sepsis in the patients included in this study. 
TABLE 5 | Mortality and Antimicrobial Drug Resistance (ADR) in p patients with sepsis, severe sepsis or septic shock admitted to Intensive Care Unit (ICU) in a Public Hospital according to antibiotic therapy, Sorocaba city, State of São Paulo, Brazil, 2013 ( $N=91$ ).

\begin{tabular}{|c|c|c|c|c|}
\hline & $\begin{array}{c}\text { Antibiotic therapy guided } \\
\text { by blood cultures } \\
\mathrm{N}(\%)\end{array}$ & $\begin{array}{l}\text { Empirical antibiotic therapy } \\
\text { (EAT) N (\%) }\end{array}$ & $\begin{array}{c}\text { Adjusted } \\
\mathrm{HR}^{\mathrm{a}}\left(95 \% \mathrm{Cl}^{\mathrm{b}}\right)\end{array}$ & $p$ value ${ }^{c}$ \\
\hline Mortality rate & $16(48.5)$ & $28(56.9)$ & $0.44(0.24-0.82)$ & 0.009 \\
\hline Antimicrobial drug resistance & 31 (93.9) & $49(84.5)$ & $0.90(0.78-1.03)$ & 0.15 \\
\hline
\end{tabular}

${ }^{a}$ HR-Hazard Ratio,

${ }^{b} \mathrm{Cl}$-Confidence interval.

${ }^{c}$ Ajusted for sex, age, Apache e Sofa.

\section{Limitations and Strength of This Study}

To our knowledge, this study adds valuable information on the management of antibiotic therapy in patients with sepsis since there are few data or recommendations that assess effective approaches to sepsis treatment in resourcelimited low-income and middle-income countries such as Brazil. The development of local studies that analyze the use of antimicrobials, as well as ADR in hospital settings, is important since the causes of sepsis and available infrastructure can be different from those described in high-income countries. It is also important to highlight that this research included the application of a robust methodology. During the entire study period, the authors made sure that the initial empirical therapy and their alteration were based on protocol established by Hospital Infection Control Committee (HICC) which monitor the use of antibiotics and resistant strains in the hospital. The local policy regarding empiric therapy was based on antimicrobial resistant patterns present in the hospital and followed the Surviving Sepsis Campaign guideline (Dellinger et al., 2013) and used the criteria of evolutionary clinicians according to Bone et al. (1992). Furthermore, this study compared two types of therapies using robust outcome measures (mortality of hospital and antimicrobial drug resistance). Data collection was prospective and cross-checked with laboratory information which was obtained directly from the laboratory's database. In addition, there was no loss of patients in this sample, all the results collected were analyzed within the original patient group and the outcomes were adjusted by confusing factors such as sex, age, APACHE II, and SOFA.

However, some limitations of our study should be acknowledged. First, the de-escalation practice was based on physician preference. Second, this study involved a small number of patients from a single public hospital. In addition, although the all patients included in this study have the possibility of de-escalation, the number of patients who had alteration of initial empirical therapy was limited. It is important to emphasize that this work was a cohort study, patients were not randomised to the two options. Thus, the de-escalation was based on clinical decision making.

Third, we initially used medical records to collect data from patients which these are not designed for research purposes. Thus, in order to reduce memory bias or lack of data in medical records any question or absence of data was requested to the team in real-time, and all variables collected were confirmed by two specialists. Finally, only patients with positive blood cultures were included in order to reduce the colonization/contamination bias due to the presence of bacteria in catheters and other access points in patients. Despite the blood culture remains the reference standard for the diagnosis of sepsis, contaminations represent up to $50 \%$ of positive blood cultures (Dargère et al., 2018). This limitation implied that, for all patients, the possibility of de-escalation existed. On the other hand, this inclusion criterion increases the specificity of the diagnosis of sepsis since the SIRS has limited specificity.

\section{Implications for Clinical Practice}

In order to improve antibiotic prescribing patterns, it is well established that accurate knowledge of the pathogens associated, and their susceptibility profile allows for a more rational selection of antibiotics for treatment of patients with sepsis. In clinical practice, however, antibiotics are often used, even when culture results are not available. The challenge for intensive care physicians is to obtain microbiological cultures before starting antimicrobial therapy (Schurink et al., 2004).

Although the early and appropriate EAT is undoubtedly an important prognostic factor in critically ill patients with sepsis, our findings clearly support the use of empirical antibiotics should be reviewed according to the susceptibility profile, when blood culture results are available. Thus, the ideal approach for the treatment of patients with sepsis, severe sepsis and septic shock admitted to the ICU should include the early administration of broad-spectrum antibiotics, together with reassessment and narrowing or discontinuation of subsequent treatment based on the results of blood cultures and antibacterial susceptibility. However, further studies are needed in order to evaluate the impact of the implementation of this practice on antibiotic resistance.

On the other hand, it is important to highlight that routine microbiological cultures and sensitivity tests may not be performed in some hospitals from most low- and middle-income countries, such as Brazil, due to a lack of personnel trained, equipment and financial resources (Taniguchi et al., 2019; Kakkar et al., 2020; Mathew et al., 2020). Without this information, clinicians do not have sufficient information to prescribe the narrowest-spectrum antibiotic needed to treat patients with sepsis. Therefore, the transferability of our findings to hospitals with more limited resources within Brazil or to other lowand middle-income countries will largely depend on the availability of laboratory support and financial resources. 


\section{DATA AVAILABILITY STATEMENT}

The raw data supporting the conclusions of this article will be made available by the authors, without undue reservation.

\section{ETHICS STATEMENT}

This study was reviewed and approved by Research Ethics Committee of the University of Sorocaba and Research Ethics Committee of the Conjunto Hospitalar de Sorocaba (CHS). The patients/participants provided their written informed consent to participate in this study.

\section{AUTHOR CONTRIBUTIONS}

JP contributed to the conception and design of the study. JP, SK, and MC collected and organized the data. LL participated

\section{REFERENCES}

Bone, R. C., Balk, R. A., Cerra, F. B., Dellinger, R. P., Fein, A. M., Knaus, W. A., et al. (1992). Definitions for sepsis and organ failure and guidelines for the use of innovative therapies in sepsis. the ACCP/SCCM consensus conference committee. american college of chest physicians/society of critical care medicine. Chest 101 (6), 1644-1655. doi:10.1378/chest.101.6.1644

Dargère, S., Cormier, H., and Verdon, R. (2018). Contaminants in blood cultures: importance, implications, interpretation and prevention. Clin. Microbiol. Infect. 24 (9), 964-969. doi:10.1016/j.cmi.2018.03.030

De Bus, L., Denys, W., Catteeuw, J., Gadeyne, B., Vermeulen, K., Boelens, J., et al. (2016). Impact of de-escalation of beta-lactam antibiotics on the emergence of antibiotic resistance in ICU patients: a retrospective observational study. Intensive Care Med. 42 (6), 1029-1039. doi:10.1007/s00134-016-4301-z

Dellinger, R. P., Levy, M. M., Rhodes, A., Annane, D., Gerlach, H., Opal, S. M., et al. (2013). Surviving sepsis campaign: international guidelines for management of severe sepsis and septic shock: 2012. Crit. Care Med. 41 (2), 580-637. doi:10. 1097/CCM.0b013e31827e83af

De Oliveira, A. C., Damasceno, Q. S., Piscoya, M., and Nicoli, J. R. (2012). Epidemiologic characteristics of resistant microorganisms present in reserves from an intensive care unit. Am. J. Infect. Contr. 40 (2), 186-187. doi:10.1016/j. ajic.2011.04.010

De Waele, J. J., Schouten, J., Beovic, B., Tabah, A., and Leone, M. (2020). Antimicrobial de-escalation as part of antimicrobial stewardship in intensive care: no simple answers to simple questions-a viewpoint of experts. Intensive Care Med. 46 (2), 236-244. doi:10.1007/s00134-019-05871-z

Denny, K. J., Gartside, J. G., Alcorn, K., Cross, J. W., Maloney, S., and Keijzers, G. (2019). Appropriateness of antibiotic prescribing in the emergency department. J. Antimicrob. Chemother. 74 (2), 515-520. doi:10.1093/jac/dky447

Engel, C., Brunkhorst, F. M., Bone, H.-G., Brunkhorst, R., Gerlach, H., Grond, S., et al. (2007). Epidemiology of sepsis in germany: results from a national prospective multicenter study. Intensive Care Med. 33 (4), 606-618. doi:10. 1007/s00134-006-0517-7

Garnacho-Montero, J., and Amaya-Villar, R. (2010). Multiresistant acinetobacter baumannii infections: epidemiology and management. Curr. Opin. Infect. Dis. 23 (4), 332-339. doi:10.1097/QCO.0b013e32833ae38b

Garnacho-Montero, J., Gutiérrez-Pizarraya, A., Escoresca-Ortega, A., CorciaPalomo, Y., Fernández-Delgado, E., Herrera-Melero, I., et al. (2014). Deescalation of empirical therapy is associated with lower mortality in patients with severe sepsis and septic shock. Intensive Care Med. 40 (1), 32-40. doi:10. 1007/s00134-013-3077-7

Gutiérrez-Pizarraya, A., Leone, M., Garnacho-Montero, J., Martin, C., and MartinLoeches, I. (2017). Collaborative approach of individual participant data of in the design of the project and drafted the manuscript. MS and FM performed the statistical analysis. MT and FF analyzed the results of antimicrobial drug resistance. All authors contributed to manuscript revision, read and approved the submitted version.

\section{FUNDING}

MC was supported by the University of Sorocaba Institutional scholarship.

\section{ACKNOWLEDGMENTS}

We are grateful to the clinical and laboratory teams from the Conjunto Hospitalar de Sorocaba (CHS) that allowed the usage of patients' information for this study.

prospective studies of de-escalation in non-immunosuppressed critically ill patients with sepsis. Expet Rev. Clin. Pharmacol. 10 (4), 457-465. doi:10.1080/ 17512433.2017.1293520

Kakkar, A. K., Shafiq, N., Singh, G., Ray, P., Gautam, V., Agarwal, R., et al. (2020). Antimicrobial stewardship programs in resource constrained environments: understanding and addressing the need of the systems. Front. Public Health. 8 , 140. doi:10.3389/fpubh.2020.00140

Kollef, M. H., Golan, Y., Micek, S. T., Shorr, A. F., and Restrepo, M. I. (2011). Appraising contemporary strategies to combat multidrug resistant gramnegative bacterial infections--proceedings and data from the gram-negative resistance summit. Clin. Infect. Dis. 53 (Suppl. 2), S33-S55. doi:10.1093/cid/ cir475

Lambregts, M. M. C., Bernards, A. T., van der Beek, M. T., Visser, L. G., and de Boer, M. G. (2019). Time to positivity of blood cultures supports early reevaluation of empiric broad-spectrum antimicrobial therapy. PloS One 14 (1), e0208819. doi:10.1371/journal.pone.0208819

Leone, M., Bechis, C., Baumstarck, K., Lefrant, J.-Y., Albanèse, J., Jaber, S., et al. (2014). De-escalation versus continuation of empirical antimicrobial treatment in severe sepsis: a multicenter non-blinded randomized noninferiority trial. Intensive Care Med. 40 (10), 1399-1408. doi:10.1007/s00134-014-3411-8

Lew, K. Y., Ng, T. M., Tan, M., Tan, S. H., Lew, E. L., Ling, L. M., et al. (2015). Safety and clinical outcomes of carbapenem de-escalation as part of an antimicrobial stewardship programme in an ESBL-endemic setting. J. Antimicrob. Chemother. 70 (4), 1219-1225. doi:10.1093/jac/dku479

Machado, F. R., Cavalcanti, A. B., Bozza, F. A., Ferreira, E. M., Angotti Carrara, F. S., Sousa, J. L., et al. (2017). The epidemiology of sepsis in brazilian intensive care units (the Sepsis PREvalence Assessment Database, SPREAD): an observational study. Lancet Infect. Dis. 17 (11), 1180-1189. doi:10.1016/ s1473-3099(17)30322-5

Martin, G. S., Mannino, D. M., Eaton, S., and Moss, M. (2003). The epidemiology of sepsis in the united states from 1979 through 2000. N. Engl. J. Med. 348 (16), 1546-1554. doi:10.1056/NEJMoa022139

Martínez, M. L., Plata-Menchaca, E. P., Ruiz-Rodríguez, J. C., and Ferrer, R. (2020). An approach to antibiotic treatment in patients with sepsis. J. Thorac. Dis. 12 (3), 1007-1021. doi:10.1177/0885066618784861

Mathew, P., Ranjalkar, J., and Chandy, S. J. (2020). Challenges in implementing antimicrobial stewardship programmes at secondary level hospitals in India: an exploratory study. Front. Public Health 8, 493904. doi:10.3389/fpubh.2020. 493904

Mathur, A. (2019). What's new in critical illness and injury science? Antibiotics in critical care: therapeutic toolbox. Int. J. Crit. Illness Injury Sci. 9 (3), 105-109. doi:10.4103/IJCIIS.IJCIIS_81_19

Paul, M., Shani, V., Muchtar, E., Kariv, G., Robenshtok, E., and Leibovici, L. (2010). Systematic review and meta-analysis of the efficacy of appropriate empiric 
antibiotic therapy for sepsis. Antimicrob. Agents Chemother. 54 (11), 4851. doi:10.1128/AAC.00627-10

Pradipta, I. S., Sodik, D. C., Lestari, K., Parwati, I., Halimah, E., Diantini, A., et al. (2013). Antibiotic resistance in sepsis patients: evaluation and recommendation of antibiotic use. N. Am. J. Med. Sci. 5 (6), 344-352. doi:10.4103/1947-2714.114165

Rhee, C., Kadri, S. S., Dekker, J. P., Danner, R. L., Chen, H.-C., Fram, D., et al. (2020). Prevalence of antibiotic-resistant pathogens in culture-proven sepsis and outcomes associated with inadequate and broad-spectrum empiric antibiotic use. JAMA Network Open 3 (4), e202899. doi:10.1001/ jamanetworkopen.2020.2899

Rudd, K. E., Johnson, S. C., Agesa, K. M., Shackelford, K. A., Tsoi, D., Kievlan, D. R., et al. (2020). Global, regional, and national sepsis incidence and mortality, 1990-2017: analysis for the global burden of disease study. Lancet 395 (10219), 200-211. doi:10.1016/S0140-6736(19)32989-7

Schurink, C. A., Hoitsma, M., Rozenberg-Arska, M., Joore, J. C., Hoepelman, I. M., and Bonten, M. J. (2004). Do cultures contribute to optimisation of antibiotic therapy in the intensive care unit? Int. J. Antimicrob. Agents 23 (4), 325-331. doi:10.1016/j.ijantimicag.2003.08.013

Sherwin, R., Winters, M. E., Vilke, G. M., and Wardi, G. (2017). Does early and appropriate antibiotic administration improve mortality in emergency department patients with severe sepsis or septic shock? J. Emerg. Med. 53 (4), 588-595. doi:10.1016/j.jemermed.2016.12.009

Silva, B. N., Andriolo, R. B., Atallah, A. N., and Salomão, R. (2013). De-escalation of antimicrobial treatment for adults with sepsis, severe sepsis or septic shock. Cochrane Database Syst. Rev. 2013 (3), Cd007934. doi:10.1002/14651858. CD007934.pub3

Singer, M., Deutschman, C. S., Seymour, C. W., Shankar-Hari, M., Annane, D., Bauer, M., et al. (2016). The third international consensus definitions for sepsis and septic shock (sepsis-3). JAMA 315 (8), 801-810. doi:10.1001/jama.2016.0287

Strandberg, G., Walther, S., Agvald Ohman, C., and Lipcsey, M. (2020). Mortality after severe sepsis and septic shock in swedish intensive care units 2008-2016-A nationwide observational study. Acta Anaesthesiol. Scand. 64 (7), 967-975. doi:10.1111/aas.13587

Tabah, A., Cotta, M. O., Garnacho-Montero, J., Schouten, J., Roberts, J. A., Lipman, J., et al. (2016). A systematic review of the definitions, determinants, and clinical outcomes of antimicrobial de-escalation in the intensive care unit. Clin. Infect. Dis. 62 (8), 1009-1017. doi:10.1093/cid/civ1199

Taniguchi, L. U., Azevedo, L. C. P., Bozza, F. A., Cavalcanti, A. B., Ferreira, E. M., Carrara, F. S. A., et al. (2019). Availability of resources to treat sepsis in Brazil: a random sample of brazilian institutions. Revista Brasileira de Terapia Intensiva 31 (2), 193-201. doi:10.5935/0103-507x.20190033

Teerawattanapong, N., Panich, P., Kulpokin, D., Na Ranong, S., Kongpakwattana, K., Saksinanon, A., et al. (2018). A systematic review of the burden of multidrug-resistant healthcare-associated infections among intensive care unit patients in southeast asia: the rise of multidrug-resistant acinetobacter baumannii. Infect. Contr. Hosp. Epidemiol. 39 (5), 525-533. doi:10.1017/ice. 2018.58

Vincent, J. L., Sakr, Y., Sprung, C. L., Ranieri, V. M., Reinhart, K., Gerlach, H., et al. (2006). Sepsis in european intensive care units: results of the SOAP study*. Crit. Care Med. 34 (2), 344-353. doi:10.1097/01.ccm. $0000194725.48928 .3 \mathrm{a}$

Von Elm, E., Altman, D. G., Egger, M., Pocock, S. J., Gotzsche, P. C., and Vandenbroucke, J. P. (2014). The strengthening the reporting of observational studies in epidemiology (STROBE) statement: guidelines for reporting observational studies. Int. J. Surg. 12 (12), 1495-1499. doi:10. 1016/j.ijsu.2014.07.013

Xie, J., Wang, H., Kang, Y., Zhou, L., Liu, Z., Qin, B., et al. (2020). The epidemiology of sepsis in chinese ICUs: a national cross-sectional survey. Crit. Care Med. 48 (3), e209-e218. doi:10.1097/ccm.0000000000004155

Conflict of Interest: The authors declare that the research was conducted in the absence of any commercial or financial relationships that could be construed as a potential conflict of interest.

Copyright (c) 2021 Pedroso, Motter, Koba, Camargo, Toledo, Del Fiol, Silva and Lopes. This is an open-access article distributed under the terms of the Creative Commons Attribution License (CC BY). The use, distribution or reproduction in other forums is permitted, provided the original author $(s)$ and the copyright owner $(s)$ are credited and that the original publication in this journal is cited, in accordance with accepted academic practice. No use, distribution or reproduction is permitted which does not comply with these terms. 